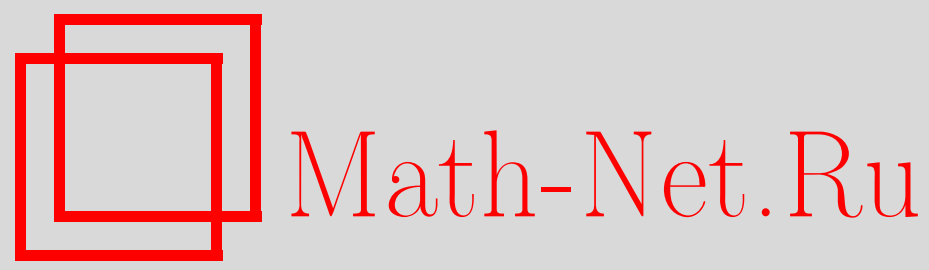

И. В. Беляков, Минимальное уклонение от нуля отображений Чебышева, соответствующих равностороннему треугольнику, Матем. заметки, 1996, том 59, выпуск 6, 919-922

DOI: https://doi.org/10.4213/mzm1791

Использование Общероссийского математического портала MathNet.Ru подразумевает, что вы прочитали и согласны с пользовательским соглашением

http://www . mathnet.ru/rus/agreement

Параметры загрузки:

IP : 3.85 .183 .62

26 апреля 2023 г., 03:06:54

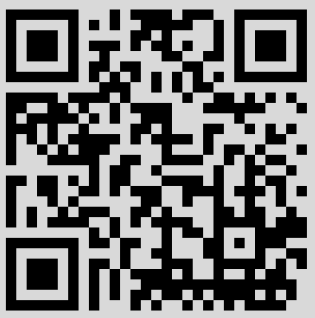




\section{МИНИМАЛЬНОЕ УКЛОНЕНИЕ ОТ НУЛЯ ОТОБРАЖЕНИЙ ЧЕБЫШЕВА, СООТВЕТСТВУЮЩИХ РАВНОСТОРОННЕМУ ТРЕУГОЛЬНИКУ}

\section{И.В. Беляков}

В работах Веселова [1], Хоффмана и Уизерса [2] была предложена конструкция, сопоставляющая афффинной группе Вейля $\widetilde{W}$ функцию $h: \mathbb{R}^{\alpha} \rightarrow \mathbb{R}^{\alpha}$, называемую обобщенным косинусом, инвариантную относительно $\widetilde{W}$ (т.е. $h(g(x))=h(x)$ $\forall g \in \widetilde{W})$ и гомеоморфную на фундаментальной области группы $\widetilde{W}$. Функция $h$ обладает полиномиальным законом преобразования:

$$
h(k x)=T_{k}(h(x)), \quad k \in \mathbb{N} .
$$

Полиномиальные отображения $T_{k}$ естественно назьвать отображениями Чебьшева, соответствующими группе $\widetilde{W}$ (см. [2]). Их свойства во многом повторяют свойства классических многочленов Чебышева. Последние соответствуют группе $\widetilde{W}\left(A_{1}\right)$, которая порождена отражениями прямой относительно концов отрезка $[0, \pi]$.

Здесь нас будет интересовать группа $\widetilde{W}\left(A_{2}\right)$, порожденная отражениями плоскости относительно сторон правильного треугольника. Обобщенньй косинус $h: \mathbb{R}^{2} \rightarrow \mathbb{C}$ в этом случае выглядит так

$$
h(u, v)=e^{2 \pi i u}+e^{2 \pi i v}+e^{-2 \pi i(u+v)},
$$

и отображает плоскость на дельтоид (которьй называют еще областью Штейнера), обозначаемый в дальнейшем через $\Delta$ (см. рис.).

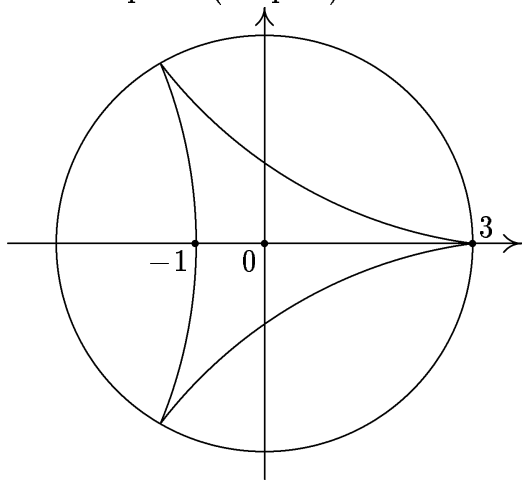

Дельтоид ограничен гипоцик лоидой с тремя ветвями, имеющей параметрическое уравнение

$$
z=2 e^{2 \pi i u}+e^{-4 \pi i u}, \quad u \in[0,1] .
$$

Отображения Чебьшшева $A_{n}$, для которых

$$
h(n u, n v)=A_{n}(h(u, v)),
$$


осуществляют $n^{2}$-кратное складывание дельтоида (об их свойствах см. [3]). Они могут быть записаны в виде многочленов от $z=h(u, v)$ и $\bar{z}$. Первые три $A_{n}$ таковы:

$$
A_{0}(z)=3, \quad A_{1}(z)=z, \quad A_{2}(z)=z^{2}-2 \bar{z} .
$$

Кроме того, справедлива рекуррентная формула

$$
A_{n}=z A_{n-1}-\bar{z} A_{n-2}+A_{n-3}, \quad n \geqslant 3,
$$

из которой видно, что степень $A_{n}$ по совокупности $z$ и $\bar{z}$ равна $n$ и главная часть равна $z^{n}$.

Договоримся, что в дальнейшем нижний индекс в обозначении многочлена будет указьвать ограничение степени по совокупности переменных, а черта над индексом будет означать, что индекс ограничивает степень по каждой переменной.

Теорема. Для многочленов $P_{n-1}(z, \bar{z})$ над $\mathbb{C}$ положим

$$
\mu=\inf _{P_{n-1}} \max _{z \in \Delta}\left|z^{n}+P_{n-1}(z, \bar{z})\right| \text {. }
$$

Тогда $\mu=3$ и достигается только при $z^{n}+P_{n-1} \equiv A_{n}$.

ДокАЗАТЕЛьство. 1) Преждевсегозаметим, что $\max _{\Delta}\left|A_{n}+c\right|>\max _{\Delta}\left|A_{n}\right|$, если $c=$ const $\neq 0$. Действительно, рассматривая неравенство $\left|A_{n}+c\right| \leqslant 3$ при $\left|A_{n}\right|=3$, получаем, что вектор $c$ должен принадлежать трем полуплоскостям, имеющим нулевое пересечение.

2) Введем переменные $\xi=e^{2 \pi i u}, \eta=e^{2 \pi i v}, \zeta=e^{2 \pi i w}$, считая $u+v+w=0$. Тогда $z=\xi+\eta+\zeta, \bar{z}=\xi \eta+\xi \zeta+\eta \zeta, A_{n}=\xi^{n}+\eta^{n}+\zeta^{n}$. Далее, $z^{n}+P_{n-1}(z, \bar{z})-A_{n}(z)=Q_{n-1}(z, \bar{z})=Q_{\overline{n-1}}(\xi, \eta, \zeta)$. Сделаем подстановку $\zeta=1 / \xi \eta$. При этом моном $\xi^{a} \eta^{b} \zeta^{c}$ перейдет в $\xi^{a-c} \eta^{b-c}$ и, значит, $Q \frac{}{n-1}(\xi, \eta, \zeta)$ перейдет в лорановский многочлен $\widetilde{Q} \overline{n-1}(\xi, \eta)$ с мономами вида $\xi^{i} \eta^{j}$, где $|i| \leqslant n-1,|j| \leqslant n-1$, а также

$$
|i-j| \leqslant n-1
$$

Итак, сделав замену переменных, получаем

$$
\mu \geqslant \inf _{\widetilde{Q} \frac{\max _{n-1}}{|\xi|=|\eta|=1}}\left|\xi^{n}+\eta^{n}+\frac{1}{(\xi \eta)^{n}}+\widetilde{Q} \frac{}{n-1}(\xi, \eta)\right|
$$

или, умножая на $\xi^{n} \eta^{n}$,

$$
\mu \geqslant \inf _{Q \frac{1}{2 n-1}} \max _{|\xi|=|\eta|=1}\left|\xi^{2 n} \eta^{n}+\xi^{n} \eta^{2 n}+1+Q \overline{2 n-1}(\xi, \eta)\right|
$$

где от $Q_{\overline{2 n-1}}$, кроме ограничения на степень, мы будем требовать только свойства (*) и равенства нулю свободного члена. (Тем самым расширяется класс возмущающих многочленов, но этого нам достаточно.)

3) Введем обозначения:

$$
\rho_{n}(\xi, \eta)=\xi^{2 n} \eta^{n}+\xi^{n} \eta^{2 n}+1, \quad f_{n}(\xi, \eta)=\rho_{n}(\xi, \eta)+Q_{\overline{2 n-1}}(\xi, \eta) .
$$


Пусть $\alpha_{1}, \ldots, \alpha_{n}-$ корни из 1 степени $n$. Известно, что

$$
\sum_{i=1}^{n} \alpha_{i}^{s}= \begin{cases}n, & \text { если } n \mid s, \\ 0, & \text { иначе. }\end{cases}
$$

Поэтому

$$
\frac{1}{n^{2}} \sum_{i, j=1}^{n} f\left(\alpha_{i} \xi, \alpha_{j} \eta\right)=\rho_{n}(\xi, \eta)+c \xi^{n} \eta^{n}
$$

(Заметим, что в силу (*) коэффициенты при $\xi^{n}$ и $\eta^{n}$ в $Q \overline{2 n-1}$ равны нулю.) Из последнего равенства

$$
\begin{aligned}
\max _{|\xi|=|\eta|=1}\left|\rho_{n}(\xi, \eta)+c \xi^{n} \eta^{n}\right| & =\max _{|\xi|=|\eta|=1}\left|\frac{1}{n^{2}} \sum_{i, j=1}^{n} f_{n}\left(\alpha_{i} \xi, \alpha_{j} \eta\right)\right| \\
& \leqslant \max _{|\xi|=|\eta|=1}\left|f_{n}(\xi, \eta)\right|
\end{aligned}
$$

Но, как отмечено в 1), при $c \neq 0$

$$
\max _{|\xi|=|\eta|=1}\left|\rho_{n}(\xi, \eta)+c \xi^{n} \eta^{n}\right|=\max _{\Delta}\left|A_{n}+c\right|>3
$$

Следователњно,

$$
\inf _{2 \frac{2 n-1}{|\xi|=|\eta|=1}}\left|\rho_{n}(\xi, \eta)+Q \overline{2 n-1}(\xi, \eta)\right|=\max _{|\xi|=|\eta|=1}\left|\rho_{n}(\xi, \eta)\right|=3 \leqslant \mu .
$$

Из определения $\mu$ видим теперь, что $\mu=3$.

4) Для доказательства единственности предположим, что для некоторого $Q \overline{2 n-1}$

$$
\max _{|\xi|=|\eta|=1}\left|\rho_{n}(\xi, \eta)+Q_{\overline{2 n-1}}(\xi, \eta)\right|=\max _{|\xi|=|\eta|=1}\left|f_{n}(\xi, \eta)\right|=3
$$

Тогда в силу пункта 1) и неравенства (1) коэффициент при $\xi^{n} \eta^{n}$ в многочлене $Q \overline{2 n-1}$ равен нулю и

$$
\rho_{n}(\xi, \eta)=\frac{1}{n^{2}} \sum_{i, j=1}^{n} f_{n}\left(\alpha_{i} \xi, \alpha_{j} \eta\right)
$$

Назовем $\left(\xi_{0}, \eta_{0}\right)$ әкстремальной точкой для $\rho_{n}(\xi, \eta)$, если $\left|\xi_{0}\right|=\left|\eta_{0}\right|=1$ и $\left|\rho_{n}\left(\xi_{0}, \eta_{0}\right)\right|=3$. Для таких точек

$$
3=\left|\rho_{n}\left(\xi_{0}, \eta_{0}\right)\right|=\left|\frac{1}{n^{2}} \sum_{i, j=1}^{n} f_{n}\left(\alpha_{i} \xi, \alpha_{j} \eta\right)\right| \leqslant \frac{1}{n^{2}} \sum_{i, j=1}^{n}\left|f\left(\alpha_{i} \xi, \alpha_{j} \eta\right)\right| \leqslant 3 .
$$

Итак, неравенство оказьвается равенством, и, значит, все (т.е. для всех $i, j$ ) $f_{n}\left(\alpha_{i} \xi, \alpha_{j} \eta\right)$ равны. Учитьвая (2), имеем $f_{n}\left(\alpha_{i} \xi_{0}, \alpha_{j} \eta_{0}\right)=\rho_{n}\left(\xi_{0}, \eta_{0}\right)$ и, в частности, $Q \overline{2 n-1}\left(\xi_{0}, \eta_{0}\right)=0$.

Таким образом, многочлен $Q \overline{2 n-1}$ должен быть равен нулю во всех экстремальных точках.

Следующие две леммы завершают доказательство. 
Лемма 1. Множсество $E_{n}$ әкстремальных точек для $\rho_{n}(\xi, \eta)$ таково:

$$
E_{n}=\left\{(\xi, \eta) \in \mathbb{C}^{2} \mid \xi^{3 n}=1, \eta^{n}=\xi^{n}\right\}
$$

В самом деле, если $|\xi|=|\eta|=1$ и $\left|\rho_{n}(\xi, \eta)\right|=3$, то $\xi^{2 n} \eta^{n}=\xi^{n} \eta^{2 n}=1$.

Лемма 2. Если $Q_{\overline{2 n-1}}(\xi, \eta)$ равен нулю на $E_{n}$, то $Q_{\overline{2 n-1}} \equiv 0$.

Пусть $Q \frac{}{2 n-1}(\xi, \eta)=\sum_{i, j=1}^{2 n-1} a_{i j} \xi^{i} \eta^{j}$.

Рассмотрим

$$
Q \frac{{ }_{2 n-1}}{(\xi, \alpha \xi)}=\sum_{k=1}^{n-2} b_{k}(\alpha) \xi^{k}, \quad \text { где } b_{k}(\alpha)=\sum_{i+j=k} a_{i j} \alpha^{j} .
$$

Из условия леммы, при $\alpha^{n}=1$,

$$
Q_{\overline{2 n-1}}(\xi, \alpha \xi)=\left(r_{n-2}(\alpha) \xi^{n-2}+\cdots+r_{1}(\alpha) \xi\right)\left(\xi^{3 n}-1\right) .
$$

Отсюда

(А) $b_{k}(\alpha)=0$ при $k=0, n-1, n, n+1, \ldots, 3 n$;

(В) $b_{k}(\alpha)+b_{k+3 n}(\alpha)=0$ при $k=1,2, \ldots, n-2$.

Случай $(\mathrm{A})$. В силу свойства $(*)$ многочлен $Q \overline{2 n-1}$ имеет члены вида $c \xi^{i} \eta^{j}$ только при $|i-j| \leqslant n-1$. Поэтому, если $i+j=$ const $=k$, то индекс $j$, как легко проверить, может принимать не более $n$ последовательных значений. Следовательно, (так как $\left.\alpha^{n+s}=\alpha^{s}\right) b_{k}(\alpha)$ - это многочлен от $\alpha$ степени $\leqslant n-1$, равный нулю во всех $\alpha_{j}$; значит, $b_{k}(\alpha) \equiv 0$.

Случай (В). Имеем

$$
b_{k}(\alpha)+b_{k+3 n}(\alpha)=\sum_{i+j=k} a_{i j} \alpha^{j}+\sum_{i+j=3 n+k} a_{i j} \alpha^{j}=0 .
$$

В первой сумме индекс $j$ меняется от 0 до $k$, во второй сумме - от $j=$ $(3 n+k)-(2 n-1)=n+k+1$ до $2 n-1$. Так как $\alpha^{n+s}=\alpha^{s}$, то и здесь мы имеем многочлен степени $\leqslant n-1$ с нулями во всех $\alpha_{j}$, а, значит, все его коэфффициенты равны нулю.

Итак, все $a_{i j}=0$, что и требовалось доказать. Теорема доказана.

Полученный результат можно распространить на отображения Чебышева, соответствующие всем группам $\widetilde{W}\left(A_{\alpha}\right)$, но об этом речь в следующих работах автора.

Автор благодарит проф. А. М. Стёпина за предложение темы исследования и внимание к работе.

Московский государственный университет

Поступило

им. М. В. Ломоносова

16.11 .95

\section{СПИСОК ЦИТИРОВАННОЙ ЛИТЕРАТУРЫ}

1. Веселов А. П. // Докл. АН СССР. 1987. Т. 292 . № 6. С. 1289-1291. 2. Hoffman M.E., Withers W. D. // Trans. Amer. Math. Soc. 1988. V. 308. №1. P. 91-104. 3. Withers W. D. // Amer. Math. Monthly. 1988. № 5. C. 399-413. 4. Веселов А. П. // УМН. 1991. Т. 46. № 5. С. 3-47. 